\title{
Valeriano y la persecución de los cristianos
}

\author{
NARCiso Santos Yanguas ${ }^{*}$
}

Poco tiempo después de la finalización de las acciones persecutorias que tuvieron como marco de actuación el reinado del emperador Decio y en el tiempo durante el que Cipriano y sus colegas trataban de restablecer el grave perjuicio moral causado a la Iglesia cristiana, el obispo de Cartago dió pruebas en repetidas ocasiones de presentimientos acerca de las posteriores persecuciones anticristianas, así como sobre el carácter cada vez más destructivo de las mismas 1.

Sin embargo, la grave tempestad que se preveía no se iba a desencadenar de inmediato, produciéndose solamente una conmoción de dimensiones reducidas en tiempos de Trebonio Galo, que se centraría casi de manera exclusiva en ciertos movimientos anticristianos, que iban a tener como escenario la capital del Imperio ${ }^{2}$.

De esta manera, por ejemplo, a través de la carta de Cipriano a Lucio contamos con noticias acerca de la confesión y proscripción de dicho personaje, así como sobre su regreso a Roma una vez elegido obispo de la ciudad para reemplazar a Cornelio, quien habría muerto como mártir en el transcurso de la persecución de los años precedentes, tal vez cuando se hallaba en el destierro ${ }^{3}$.

El relato de Cipriano sobre esta fase de persecuciones en Roma parece hallarse de acuerdo con el hecho de que Dionisio de Alejandría afirma que

Universidad de Oviedo.

Cypr., Epist. 57.1-2 y 5, y 58.1-2, 8 y 11.

Epist. 60.1-2. Cf. N. SANTOS, "Decio y la persecución de los cristianos" (en prensa).

3 Epist. 61. Cf. P. KeRESzTES, "Two Edicts of the Emperor Valerian", VChr 29 (1975), 81. 
Galo aprobaba algunas medidas represivas contra los cristianos, en especial las que tenían como objetivo desterrar a los hombres santos de la Iglesia ${ }^{4}$.

Sin embargo, estas noticias no constituyen más que pruebas de escaso valor para poder suponer que en estos momentos tendría lugar un serio hostigamiento contra las comunidades cristianas en cualquier parte del Imperio, ni incluso en el lugar elegido como refugio por el propio Cipriano ${ }^{5}$.

De una forma o de otra el emperador Trebonio Galo contaría con el poder en sus manos solamente a lo largo de un breve período de tiempo ${ }^{6}$; en este sentido sabemos que una plaga de efectos devastadores, que tendría su origen en torno al año 252, perduraría varios años, viéndose envuelto el propio emperador en sus efectos mortales, por lo que sería reemplazado en el trono a mediados de junio del año siguiente por Emiliano, gobernador de Mesia inferior, a quien sucedería igualmente poco tiempo después Valeriano, quien con anterioridad había desempeñado las funciones de censor durante el reinado de Decio ${ }^{7}$.

Se puede afirmar, por consiguiente, que el edicto emitido en tiempos de Trebonio Galo pudo haber quedado reducido a una orden estrictamente local con el fin de hacer cumplir los sacrificios a las divinidades paganas ${ }^{8}$, mientras que las referencias premonitorias y visionarias de Cipriano se hallarian relacionadas con la fase de persecución anticristiana llevada a cabo en tiempos de Valeriano, incluyendo dentro de la misma su propio destierro y martirio, al igual que una serie de sufrimientos que alcanzarian al conjunto de fieles a cuya cabeza se encontraba como director de sus anhelos religiosos ${ }^{9}$.

De esta manera tanto a Cipriano, en cuanto obispo, como a la Iglesia cristiana en su conjunto se les permitiria llevar a cabo durante estos años la reconstrucción de las ruinas (tanto espirituales como físicas) de las comunidades cristianas, disfrutando durante 4-5 años de un periodo de paz cuando menos relativa ${ }^{10}$.

\footnotetext{
4 Euseb., HE 7.1. Cf. J. MOLTHAGEN, Der römische Staat und die Christen im zweiten und dritten Jahrhundert, Gotinga 1975 ( $2^{\mathrm{a}}$ edic.), p. 85.

5 G. SCHÖNAICH (Die libelli und ihre Bedeutung für die Christenverfolgung des Kaisers Decius, Goglau 1910, p. 11), nos da cuenta de la existencia de una orden general de sacrificar extensiva a toda la población de las provincias romanas basándose en el edicto proposito de Cipriano (Epist. 59.6).

6 H. MAtTINGLY, "The Reigns of Trebonius Gallus and Volusian", NC (1946), 136 y ss.

7 G.M. BERSANETTI, "Valeriano ed Emiliano", RFIC 77(1948), 257 y ss.

A. ALFOLDI, "ZU den Christenverfolgungen in der Mitte des 3. Jahrhunderts", Klio 31(1938),

9 Sobre todos estos problemas remitimos a $\mathrm{Ch}$. SaUmagne, Saint Cyprien, évêque de Carthage, papa d'Afrique (248-258), Paris 1975.

10 P. Keresztes, "Two Edicts of the Emperor Valerian", p. 82.
} $337-338$ 
En cuanto a Dionisio de Alejandría, por una parte se refiere con cierta profusión de detalles a la plaga que tendría lugar en época postvaleriana en los siguientes términos:

"Y después la gente se admira y esta insegura de dónde proceden las continuas pestes y las graves enfermedades, asi como las corrupciones de toda clase y la reiterada y variada mortandad de los hombres, y por qué la gran ciudad no sostiene ya por si misma aquella multitud tan grande de hombres que antes alimentaba, desde los niños de pecho hasta los ancianos de extrema vejez, pasando por el enorme número de viejos prematuros, como se les llamaba" ${ }^{11 .}$

Sin embargo, no parece afirmar nada acerca de los efectos de la misma durante el período que siguió al emperador Decio ${ }^{12}$. Junto a ello Cipriano nos ofrece una dramática descripción acerca de las consecuencias que esta misma plaga provocaría en el territorio norteafricano ${ }^{13}$.

Ante la nueva situación creada, y al margen de una persecución más o menos cercana, había que contar con el peligro inmediato de los abocados al martirio o, cuando menos, a la muerte, muchos de los cuales serian cristianos que con anterioridad habían apostatado pero que se hallaban dispuestos en estos momentos a dar los pasos necesarios para su readmisión en el seno de la Iglesia y su rehabilitación como miembros efectivos de las comunidades de fieles ${ }^{14}$.

En otros términos, la Iglesia se veía en la necesidad de negociar con un amplio número de apóstatas (lapsi), quienes, movidos claramente por el peligro real de muerte que les acechaba o por un simple y sincero arrepentimiento, buscaban volver al seno de la comunidad cristiana con la mayor premura ${ }^{15}$.

Por consiguiente, al margen de la ampulosa afirmación de Porfirio en el sentido de que una cantidad muy elevada de cristianos moriria en el transcurso de las persecuciones de los emperadores Decio y Valeriano ${ }^{16}$, el número que conocemos en la actualidad, tanto de mártires como de

"Euseb., HE 7.21.9. Cf. 7.22 y M. SORDI, "Dionigi d'Alessandria, Commodiano ed alcuni problemi della storia del III secolo», Atti della Pontificia Accademia Romana di Archeologia Ser. $3^{\text {a }}$ 35 (1963), 126 y ss.

12 Euseb., HE 7.11.24.

13 De mortalitate 15-16.

i4 A.A. EHRHARDT, "Cyprian, the Father of Western Christianity", ChQRev 133 (1941-1942), 178 y ss.

${ }_{15}$ Cypr., Epist. 30.3; 55.13.14 y 17; De lapsis 27-28. Cf. P. Keresztes, "The Decian libelli and Contemporary Literature", Latomus 34 (1975), 773-774.

${ }_{16}$ Porphyr., fragm. 36. Cf. Euseb., HE 6.41-42. 
confesores, parece haber sido muy inferior, especialmente si lo comparamos con el enormemente extenso de quienes se habian convertido en apóstatas $^{17}$.

Como consecuencia de la nueva situación creada la devastación de la Iglesia cristiana alcanzaría unos caracteres espantosos ${ }^{18}$, de manera que un número significativo de obispos y sacerdotes, al igual que conocemos por ejemplo en el caso de Cipriano, se verían obligados a buscar un lugar seguro para salir indemnes de las amenazas y peligros que sobre ellos se abatían ${ }^{19}$, al tiempo que una cantidad incontable de cristianos seguirian el ejemplo de sus pastores, marchando al destierro muchos de ellos ${ }^{20}$ y siendo capturados en ocasiones por diversas hordas de bárbaros que habitaban las regiones en las que recalaban ${ }^{21}$.

A pesar de ello una gran multitud de cristianos, en realidad la mayoría de ellos, desfallecerían, pasando a convertirse en apóstatas, teniendo que incluir entre los mismos incluso a ciertos miembros del clero y a algunos obispos ${ }^{22}$. En este sentido el mismo Cipriano se refiere en ocasiones a los denodados esfuerzos de algunos de tales personajes, así como a los millares de solicitudes realizadas en su favor con el único objetivo de lograr su pronta readmisión en el seno de la comunidad cristiana ${ }^{23}$.

Todo este conjunto de esfuerzos por parte de quienes habian desfallecido ante las presiones de las autoridades romanas, unido a la respuesta dada por las jerarquias eclesiásticas, culminaría, en el transcurso de los pacíficos años de reconstrucción, en la gran controversia entablada en torno a las condiciones y circunstancias de la readmisión de dichos individuos en el seno de la Iglesia, originándose incluso algunos cismas, como por ejemplo el encabezado por Novaciano ${ }^{24}$.

Los diferentes tipos de apostasia han sido definidos y clasificados con sumo cuidado por el propio Cipriano en varios pasajes de sus escritos ${ }^{25}$,

17 Cypr., Epist. 9-12;19-20; 22; $24 ; 38-40 \ldots$

18 Cypr., Epist. 9.2 y 30.5. Cf. Ch. SAUMAGne, "La persécution de Dèce à Carthage d'aprés la correspondence de saint Cyprien", BSAF (1957), 23 y ss.

19 Cypr., Epist. 14.2; 30.8...

20 Cypr., Epist. 14.4

21 Euseb., HE 6.42.2-4. Cf. Cypr., Epist. 62 y G.W. ClaRke, "Barbarian Disturbances in North Africa in the Mid-Third Century", Antichton 4 (1970), 78 y ss.

22 Cypr., Epist. $14.1 ; 55.1$ y $10 ; 56.1 ; 59.11$ y $18 ; 65$ y 67

23 Cypr., Epist. 20.2; 30.2-3; 44.45 y 50.

24 Euseb., HE 7.2-9. Cf. D.S. WALLACE-HadRILL, "The Eusebian Chronicle», JThS N.S. 6 (1955), 248 y ss.

${ }^{25}$ Epist. 30.3; 55.13-14 y 26; 56.1-2; De lapsis 8; $27-28$ y 30. Cf. G.W. Clarke, "The Epistles of Cyprian", Essays E.M. Blaiklock, Auckland 1970, pp. 48 y ss. 
habiendo logrado determinar al mismo tiempo con exactitud las condiciones necesarias en cada caso para su readmisión en el seno de la comunidad cristiana ${ }^{26}$.

Con la finalidad de poder debatir éstas y otras muchas cuestiones vinculadas a ellas, tales como el bautismo por segunda vez, la pérdida del ejercicio del sacerdocio o los cismas, se celebrarian algunos sínodos de obispos, en los que la concurrencia fue muy amplia ${ }^{27}$.

Simultáneamente tendrían lugar abundantes encuentros imprevistos y frecuentes contactos epistolares entre obispos de regiones muy distintas y distantes entre sí, extendiéndose incluso esta correspondencia epistolar a los propios papas, con la finalidad de poder resolver los extremadamente serios problemas planteados a la Iglesia por este período de paz previo al desencadenamiento de la gran persecución de Valeriano ${ }^{28}$.

La tormenta desencadenada en tiempos de dicho emperador pondria fin durante cierto tiempo a las controversias eclesiásticas del período de paz precedente. No obstante, la gran labor de readmisión de los apóstatas contaria, de acuerdo con Cipriano, con la preparación de la multitud dispuesta a afrontar la muerte y la persecución como cristianos maduros; de esta manera, si tenemos presente su proceder durante los años siguientes, encontraremos que en realidad se hallaban ya suficientemente preparados para ello ${ }^{29}$.

Teniendo en cuenta la severidad alcanzada por la persecución de la Iglesia en tiempos de Valeriano puede parecer extraño que el reinado de dicho emperador hiciera frente a los adeptos del cristianismo tras un período de paz tan sorprendente para ellos. Si hemos de creer las noticias de Dionisio de Alejandría ${ }^{30}$, la razón estribaría en su disposición excepcionalmente favorable con respecto a los cristianos.

Además de un cierto número de cartas de Cipriano y algunas Actas y Pasiones de mártires aprovechables desde el punto de vista histórico, las

Epist. 57.1. Cf. Epist. 54.3-4y 55.6-7.

27 Cypr., Epist. $57.1-2 ; 64 ; 67 ; 70$ y 72 . Cf.G. KLEIN, "Die hermeneutische Struktur des Kirchengedankes bei Cyprian", ZKG 68 (1957), 48 y ss.

28 Cypr., Epist. 75; Euseb., HE 7.2-9...

${ }^{29}$ P. Keresztes, "Two Edicts of the Emperor Valerian", p. 83.

30 Euseb., HE 7.10.1. 
noticias más apropiadas para conocer las medidas anticristianas tomadas por Valeriano pueden hallarse en la Historia Eclesiástica de Eusebio de Cesarea ${ }^{31}$. La supervivencia del obispo de Alejandría, tanto a la persecución de Decio como a la de Valeriano, causa cierta perplejidad a algunos historiadores actuales, resultando sin embargo mucho más extraño aún el caso de uno de sus compañeros obispos, un tal Germano ${ }^{32}$ : en su apología (pro vita sua) aporta, frente a las sospechas de este Germano, una explicación muy razonable, aunque igualmente prodigiosa, de su supervivencia a lo largo de todos estos años ${ }^{33}$.

Las medidas que aparecerían recogidas en el actualmente perdido primer edicto de Valeriano, promulgadas en una fecha avanzada del verano del año 257, pueden ser distinguidas con bastante claridad en el relato de Dionisio de Alejandría correspondiente a su defensa contra Germano ${ }^{34}$. En unos términos muy similares vamos a encontrar redactadas estas mismas causas en el juicio que se llevaría a cabo contra Cipriano ${ }^{35}$.

De las afirmaciones de dichas fuentes parece desprenderse evidentemente, resultando ser un hecho aceptado por parte de la historiografía actual en buena medida, que el emperador ordenaría a los componentes de la jerarquía eclesiástica que reconocieran los ritos romanos mediante su participación en los mismos ${ }^{36}$ y que los cristianos en su conjunto no celebraran asambleas ni visitaran los lugares de enterramiento bajo la amenaza de pena capital ${ }^{37}$.

A pesar de las órdenes imperiales en el sentido de que todos los habitantes del Imperio que no siguieran la religión romana estaban obligados a reconocer los ritos paganos ${ }^{38}$, resulta totalmente claro que, a partir de las noticias de Dionisio de Alejandría y de Cipriano, únicamente los integrantes de la jerarquía de la Iglesia serian emplazados por sus gobernadores

${ }^{31}$ HE 7.10-13. Cf. W. VOELKER, "Von welchen Tendenzen sich Eusebius bei Abfassung seiner Kirchengeschichte leiten?", VChr 4 (1950), 157 y ss., y G. LAzZAT, "Nota su Eusebio epitomatore di Atti di martirì, Studi Calderini-Paribeni, Milán 1956, 1, pp. 377 y ss.

32 G.T. OвORN, "Why did Decius and Valerian proscribe the Christians?", ChHistory 2 (1933), 67 y ss.

${ }^{33}$ Euseb., HE 6.40.1-9. Cl. 7.11.1-26.

34 HE 7.10.1-4. Cf. 7.10.5-9 y B. KOENING-OCKENFELS, "Christliche Deutung der Weltgeschichte bei Euseb von Cäsarea", Saeculum 27 (1976), 350 y ss.

${ }_{35}$ Acta proconsularia sancti Cypriani 1. Cf. Cypr., Epist. 76-79.

36 Acta proconsularia sancti Cypriani 1.1-2 y Euseb., HE 7.10.3-9.

${ }^{37}$ Acta proconsularia sancti Cypriani 1.3 y ss., y Euseb., HE 7.11 .4 y 10-11. Cl. J. MOLTHAGEN, Der römische Staat und die Christen im zweiten und dritten Jahrhundert, pp. 87-90.

${ }_{38}$ F. MuLLAR, "The Imperial Cult and the Persecutions", Le culte des souveraines dans l'empire romain, Vandoeuvres-Genèves, $19873, \mathrm{pp} .143$ y ss. 
respectivos para venerar a los dioses y que como castigo único les sería impuesto el destierro por haber rehusado cumplir dicha orden ${ }^{39}$.

De este hecho parece desprenderse que las previsiones del edicto, aunque considerasen la persecución de los laicos como una posibilidad, no desembocarian en una forma invariable de actuación por parte de la administración romana en las diferentes regiones y provincias del Imperio ${ }^{40}$. En realidad la correspondencia de Cipriano relativa a estos hechos nos muestra que no sólo los obispos, sacerdotes y diáconos (es decir la jerarquía eclesiástica de las comunidades cristianas) sino también los laicos cristianos serian condenados a trabajar en las minas ${ }^{41}$.

En este sentido, además de resultar evidente que los laicos cristianos, incluyendo entre ellos a mujeres y niños, en compañía de numerosos obispos, sacerdotes y diáconos númidas, serían condenados a las labores mineras en la región de Numidia ${ }^{42}$, parece igualmente interesante destacar que algunos componentes de las órdenes menores, en concreto un subdiácono y varios acólitos, junto con otros cristianos fueron a visitar a los prisioneros y a llevarles provisiones sin ser molestados al parecer para nada por ello ${ }^{43}$.

La presencia de laicos cristianos entre los clérigos condenados a los trabajos de las minas, aunque en raras ocasiones, pudo producirse de una forma totalmente normal; en realidad dichas personas habrían confesado públicamente su cristianismo ante el tribunal y el gobernador correspondiente, quien únicamente se mostraba dispuesto a enviar a algunos a las minas y a entregar a otros a la muerte.

La inspiración para estas personas parece haberla constituido la confesión y destierro de Cipriano, tal vez de forma totalmente inesperada ${ }^{44}$ : la nueva imagen del hasta entonces tímido obispo de Cartago llegaría a convertirse en esos momentos, y con posterioridad durante bastante tiempo, en fuente de inspiración para los cristianos de Africa y Numidia. Como consecuencia de ello los seglares cristianos pudieron pasar a ser víctimas

39 P. KEREsztes, "Two Edicts of the Emperor Valerian", p. 84.

40 Th. PÉKARY, "Bemerkungen zur Chronologie des Jahrzehnts 250-260 n..Chr.", Historia 11 (1962), 123 y ss.

41 Epist. 76. Ver igualmente Epist. $77-79$

42 Cypr., Epist. 76 y 78

${ }^{43}$ Cypr., Epist. 77.3.2: “Además, con nuestro queridisimo Quirino nos habeis enviado, por medio del subdiácono Herennio y los acólitos Lucano. Máximo y Anastasio, recursos para distribuir y proveer asi a nuestras necesidades".

${ }_{44}$ Cypr., Epist. 76.5; 77.2 y 78.1. Cf. E.L. HummeL, The Concept of Martyrdom according to St. Cyprian of Carthage, Washington 1946. 
de este primer edicto de persecución, que los dejaría libres de cualquier sospecha en el caso de que no siguieran celebrando asambleas y se mantuvieran alejados de sus lugares de enterramiento ${ }^{45}$.

\section{III}

La extraordinariamente sangrienta persecución, al menos en teoría, provocada como resultado del primer edicto de Valeriano permaneceria inalterable en su esencia hasta mediados del año 258 , momento en el que vería la luz un segundo documento con la finalidad de no dejar salida a los numerosos puntos de escape presentados por el primero: el contenido de tal escrito, obra igualmente de dicho emperador, que se nos ha perdido al igual que el primero, se halla referido de forma extraordinariamente simple en la carta 80 de Cipriano ${ }^{46}$.

Este documento epistolar constituye un relato detallado acerca de un obispo, Succeso, y esta basado en una averiguación llevada a cabo por ciertos espias de Cipriano enviados a la capital del Imperio con el fin de investigar los rumores que se habian extendido en Cartago, e indudablemente también en Roma, referentes a las nuevas medidas imperiales que ya se habian puesto en práctica febrilmente en la ciudad romana, al menos desde comienzos de agosto del año $258{ }^{47}$.

Las medidas recogidas por Cipriano que formarian parte con toda certeza del contenido del nuevo decreto anticristiano serian las siguientes:

a) que los obispos, sacerdotes y diáconos deberían ser entregados a la muerte con toda rapidez;

b) que los senadores, personajes de elevada posición y caballeros romanos se verían privados de sus rangos sociales y bienes patrimoniales, y en el caso de que perseverasen como cristianos deberían sufrir un castigo capital;

c) que las matronas romanas perderían todos sus bienes y serian enviadas al destierro; $y$

d) por último, que aquellos empleados de la administración imperial que hubiesen confesado con anterioridad su cristianismo o lo hicieran en

45 J. MOLTHAGEN, Der römische Staat und die Christen im zweiten und dritten Jarhundert, pp. 91-92.

46 Epist. 80.1. Cf. G.W. ClaRkE, “Prosopographical Notes on the Epistles of Cyprian, III: Rome in August, 258», Latomus 34 (1975), 437.

47 P.KERESZTES, "Two Edicts of the Emperor Valerian", p. 85. 
estos momentos verían confiscados sus bienes y entregados, en hipoteca, al patrimonio imperial.

Además de este relato de Cipriano contamos con otra serie de documentos que testimonian algunas de las medidas anteriormente mencionadas: así, en la misma carta el obispo de Cartago refiere la muerte del obispo de Roma, Sixto, y de cuatro de sus diáconos, ocurridas el día 6 de agosto del $258{ }^{48}$, al tiempo que la muerte del propio Cipriano nos aparece relatada en las Actas de su martirio ${ }^{49}$.

En ambos casos podemos encontrar, en sus líneas generales, elementos acordes con las primeras medidas puestas en práctica tras la promulgación del segundo de los edictos. Por otro lado la Pasión del obispo Fructuoso de Tarragona y de sus dos diáconos Augurio y Eulogio nos ofrece pruebas similares, aunque indica igualmente que los clérigos de rango inferior, al igual que los cristianos laicos, quedarían libres ${ }^{50}$.

Por lo que respecta a la Pasión de los obispos Agapio y Secundino, del diácono Santiago, del lector Mariano y de un buen número de cristianos seglares, contamos con una información histórica más apropiada, que nos confirma algunas de las medidas que encerraba este segundo edicto imperial ${ }^{51}$. El hecho de que los obispos Agapio y Secundino fueran llamados desde el lugar a que habían sido desterrados con ocasión del primer edicto se halla totalmente de acuerdo con la llamada que el propio Cipriano recibiría desde Curubis con vistas a su regreso ${ }^{52}$.

Al igual que en el caso de Cipriano en Cartago, los personajes anteriormente mencionados serian entregados a la muerte por el gobernador de Numidia, cumpliendo de esta manera las órdenes contenidas en el segundo decreto de Valeriano. Por su parte el arresto y ejecución de los clérigos de rango inferior nos viene corroborado igualmente por las referencias de Cipriano a dicho edicto ${ }^{53}$.

48 Epist. 80.1 y 4. Cf. P. CORSSEN, "Der Schauplatz der Passion des römischen Bischofs Sixtus ॥», ZNThW $16(1915), 147$ y ss.

Acta proc. s. Cypriani $2 \cdot 5$.

5o Passio Sanctorum Martyrum Fructuosi episcopi, Auguri et Eulogi diaconorum 1-2. Sobre dicho documento cf. H. Musurillo, Acta Martyrum. The Acts of the Christian Martyrs, Oxford 1972, pp. 176 y ss.

5. Passio Sanctorum Mariani et lacobi, en H. Musurillo, The Acts of the Christian Martyrs, pp. 194 y ss.

52 Sobre estos problemas remitimos a P. PASCHINI, "La persecuzione di Valeriano", StudRom 6 (1958), 130 y ss.

53 Epist. 80. Cf. Passio Sanctorum Mariani et lacobi 4. 
En realidad parece haber sido bastante significativo el número de clérigos entregados a la muerte con motivo de esta actuación ${ }^{54}$; la información más sobresaliente de esta Pasión la constituye sin duda el hecho de que un personaje de rango ecuestre fuese encarcelado igualmente como consecuencia de su confesión del cristianismo, siendo condenado a muerte posiblemente después por ello ${ }^{55}$.

De esta manera, aunque el relato de Cipriano acerca del segundo edicto imperial deja sin mencionar al conjunto de los cristianos laicos ${ }^{56}$, es posible afirmar que las medidas a ellos concernientes y que aparecian descritas en el primero de los edictos aún continuarían vigentes. Por ello resulta fácil suponer, de acuerdo con el documento hagiográfico a que nos venimos refiriendo, que la suerte de los laicos cristianos pudo haber estado vinculada a la celebración o no de sus asambleas religiosas ${ }^{57}$.

Por otro lado la Pasión de Montano, Lucio y otros compañeros mártires corrobora igualmente el cumplimiento estricto de las medidas correspondientes al clero ${ }^{58}$ : todos los sacerdotes y diáconos cristianos deberían ser entregados a la muerte en el caso de que perseverasen en su fe, mientras que los investidos con las órdenes inferiores serian dejados en libertad.

Sin embargo, el castigo de numerosos cristianos laicos a manos del entonces gobernador de Cartago a principios del año 259 contrasta extraordinariamente con el trato dispensado por su predecesor con motivo del juicio de Cipriano unos meses antes: la explicación de este hecho puede encontrarse en que dichos seglares cristianos habrían sido apresados mientras celebraban asambleas, por lo que no serian ciertamente victimas de una persecución indiscriminada ${ }^{59}$.

Mientras que la existencia del primer edicto de Valeriano aparece extraordinariamente clara en las referencias de Dionisio de Alejandría a las

54 Passio Sanctorum Mariani et lacobi 10-11. Cf. W.H.C. FREND, Martyrdom and Persecution in the Early Church, Nueva York 1967, pp. 315-323.

55 Passio Sanctorum Mariani et lacobi 8-9. Cf. P. KereszTES, “Two Edicts of the Emperor Valerian", pp. 85-86.

56 Epist. 80. Cf. G.W. ClaRke, "Prosopographical Notes on the Epistles of Cyprian, Ill: Rome in August, 258 ", pp. 437 y ss.

57 Passio Sanctorum Mariani et lacobi 4. Cf. J. Molthagen, Der römische Staat und die Christen im zweiten und dritten Jahrhundert, pp. 92-93.

58 Passio Sanctorum Montani et Lucii en H. MusurllLo, Acta Martyrum. The Acts of the Christian Hartyrs, pp. 214 y ss.

59 Passio Sanctorum Montani et Lucii 9, 13 y 14. Cf. J. VOGT, "Christenverfolgungen", RAC 2 (1954), 1187-1188. 
medidas tomadas por parte de dicho emperador con respecto a los cristianos ${ }^{60}$, la presencia del segundo de ellos no se refleja en ninguna parte de las mismas de forma explicita.

Por ello es posible que radique ahí precisamente la causa por la que el obispo nunca seria juzgado por su vida tras la proclamación del segundo edicto, a pesar de que pudiera haberlo sido por ello.

Aun en este caso los indicios y síntomas del nuevo edicto mortal es posible reconocerlos en la defensa un tanto precipitada que aparece relatada en Dionisio acerca de su conducta en el transcurso de las persecuciones de Decio y Valeriano: se trata de noticias explicitas, aunque en ocasiones no muy significativas ${ }^{61}$, referentes a las persecuciones que tuvieron como escenario la ciudad de Alejandría y sus alrededores durante el reinado de Valeriano.

A ello hemos de añadir que los relatos del martirio de los clérigos y laicos cristianos pueden ser considerados como hechos resultantes de la puesta en funcionamiento del segundo decreto por parte del gobernador de dicha ciudad ${ }^{62}$. En este sentido algunos componentes del clero marcharían a ocultarse entre la abigarrada población de la propia Alejandría, mientras que otros, mejor conocidos por el conjunto de la población, abandonarían la ciudad o irían a esconderse en territorio egipcio ${ }^{63}$.

Resulta particularmente significativo a este respecto tener en cuenta que, de acuerdo con las órdenes emanadas del segundo de dichos decretos, los clérigos serían llamados, con el fin de ser juzgados nuevamente y dar así cumplimiento al segundo edicto, de los lugares a que ellos mismos se habian desterrado cumpliendo las normas del primer decreto ${ }^{64}$.

Otros, como en el caso de Cipriano, serian reclamados desde el exilio y establecidos en sus anteriores lugares de asentamiento, sin duda con el objetivo de tenerlos próximos a los tribunales en el caso de que se hiciera necesario cumplir las normas del segundo decreto ${ }^{65}$. Esto mismo debió

\footnotetext{
60 Euseb., HE 7.11.1-7.

6) Euseb., HE 7.11.24-25.

62 A. RousSELLE, “La persécution des chrétiens à Alexandrie au III siècle", $R D 52$ (1974), 243 y ss.

63 Es posible que no se tratara de integrantes del clero sino de componentes del colectivo de altos personajes (egregii viri), a quienes hace referencia el segundo edicto (Cypr., Epist. 80.1).

64 Passio Sanctorum Mariani et lacobi 2-3. Cf. P. KeRESzTES, "Two Edicts of the Emperor Valerian", p. 87.

65 Acta proc. s. Cypriani 2. Cf. Passio Fructuosi 1 y Passio Sanctorum Mariani et lacobi $1-2$.
} 
sucederle a Dionisio de Alejandria, quien se esfuerza en explicar los hechos en los que se vio envuelto y el desarrollo de los mismos para poder sobrevivir a la persecución de Valeriano: de acuerdo con sus propias noticias se le ordenaria trasladarse desde su lugar de destierro hasta Colluthion, población cercana a la ciudad de Alejandría, para que él y sus compañeros pudieran ser arrestados con facilidad en el momento en que los gobernadores trataran de buscarlos ${ }^{66}$.

Como consecuencia de ello, un día cualquiera este obispo y cuatro de sus acompañantes, posiblemente miembros del clero, serian arrestados nuevamente por un centurión y una compañía de soldados ${ }^{67}$. No obstante, antes de ser llevados a presencia del gobernador de Alejandría para ser juzgados por sus vidas, serian puestos en libertad y, contra el deseo de Dionisio, conducidos por algunos simpatizantes cristianos del lugar a una zona desierta mucho más alejada.

De este hecho parece desprenderse que el obispo de Alejandría no tuvo ocasión de defender su vida ante el tribunal siguiendo las pautas del segundo edicto y de morir como un mártir como era su deseo ${ }^{68}$.

Por consiguiente lograría sobrevivir a esta persecución, que continuaría teniendo vigencia a lo largo de todo el año 259 , en concreto hasta el momento en que el emperador sería hecho prisionero y muerto por los persas en los meses intermedios del año siguiente ${ }^{69}$.

Con respecto a estos hechos se pueden hacer abundantes observaciones, en especial en lo que se refiere at relato de Dionisio de Alejandria que explica la persecución de la Iglesia llevada a cabo por Valeriano: en su carta dirigida a Hermamon, poco antes de explicar la persecución incoada por dicho emperador, nos menciona la existencia de una era de paz hasta entonces desconocida para los cristianos, que se extenderia igualmente durante los primeros años del reinado de Valeriano, paz que hace sumamente difícil comprender las medidas tomadas en la posterior etapa de persecución ${ }^{70}$.

De esta manera, parece fuera de toda duda que, en una primera fase, aproximadamente en el transcurso de los cuatro primeros años de este

66 Euseb., HE 7.11.14-17. Se hallarian en una situación que podemos calificar como de “libertad vigilada". Cf. H. GRÉGOIRE y otros, Les persécutions dans l'empire romain, Bruselas 1964 , pp. 49-50.

67 Euseb., HE 7.11.22-23.

68 El ejemplo de tres mártires cristianos en Cesarea de Palestina se refiere igualmente a este problema. Ver, además, Euseb., HE 7.12.1.

69 G. WALSER y Th. PÉKARY, Die Krise des römischen Reiches, Berlín 1962, pp. 28 y ss.

70 Euseb., HE 7.10.2-3 y 22-23. 
reinado, los cristianos gozarían de un periodo de paz excepcional; sin embargo, la situación cambiaría con relación al estilo utilizado en sus afirmaciones por Dionisio, quien se muestra excesivamente retórico en cuanto a su descripción de la amistad de Valeriano con respecto a los cristianos, apareciendo al mismo tiempo demasiado indulgente en ciertas expresiones desorbitadas ${ }^{71}$.

\section{IV}

Este periodo de tiempo se nos muestra excepcionalmente importante a la hora de compararlo con la labor destructura y desintegradora que llevó a cabo la persecución de Decio contra la Iglesia cristiana ${ }^{72}$. Sin embargo, podemos pensar que no serian menores las consecuencias que se derivarian durante el reinado de Valeriano para la propia Iglesia: los términos en que se expresa el obispo de Alejandria se hallan justificados realmente por el hecho de que el segundo decreto de Valeriano toma una serie de medidas, hasta entonces desconocidas, contra los senadores y altos personajes cristianos, así como contra los adeptos del cristianismo pertenecientes al orden ecuestre y las matronas y oficiales de la administración imperial, partidarios igualmente de dicha doctrina ${ }^{73}$.

Aun cuando, a primera vista, no parece digno de crédito el hecho de que todas estas personas de rango superior integradas en el gobierno y sociedad romanos hubieran abrazado la religión cristiana durante estos años de paz del reinado de Valeriano, podemos asegurar que, en el momento en que se tomó esta medida excepcional contra ellos, tales individuos se sintieron seguros para ser considerados como cristianos, a pesar de que su optimismo seria objeto de prueba demasiado pronto.

Como consecuencia de ello, esta situación tan incuestionable de paz, hasta entonces desconocida y tan favorable a los cristianos, comporta una dificultad más ardua a la hora de explicar el improvisado comienzo de la persecución en tiempos de dicho emperador, sin tener en cuenta el hecho de que el obispo de Alejandría nos ofrece una explicación muy simple al respecto: la causa del desencadenamiento de tal persecución estribaría

74 P. KERESzTES, "Two Edicts of the Emperor Valerian», p. 88.

72 Ver, entre otros, O. Gióndano, I cristiani nel III secolo. L'editto di Decio, Msnina 1967; P. KERESzTES, "The Decian libelli and Contemporary Literature", pp. 761 y ss., y G.W. CLARKE, "Some Observations on the Persecution of Decius", Antichton 3 (1969), 63 y ss.

${ }_{73}$ W. ECK, "Das Eindringen des Christentums in den Senatorenstand bis zu Konstantin d. Gr.", Chiron 1 (1971), 381 y ss. 
en el hecho de que Macriano, movido por su odio con respecto a los cristianos, habría persuadido a Valeriano para que los persiguiera y diera muerte ${ }^{74}$.

Por otro lado la afirmación de Dionisio acerca de la implicación de Macriano en las prácticas ocultas egipcias, así como de los artilugios utilizados por él para envolver al emperador en las mismas ${ }^{75}$, puede resultarnos una historia un tanto extraña, aunque en modo alguno puede ser considerada como algo descabellado, de acuerdo con lo que piensan algunos historiadores de nuestros días ${ }^{76}$.

Así pues, no podemos entender como una mera ficción de Dionisio el hecho de que Macriano fuera un enemigo destacado de la Iglesia y se convirtiera por ello en responsable de la persecución de los cristianos. Es más, dicho autor utiliza su retórica con el objetivo de trasladar el énfasis de la persona de Valeriano a la de Macriano con respecto a la responsabilidad de la persecución, de manera que el emperador se nos muestra como un personaje que nunca podía haber perseguido a la Iglesia sin la existencia de una influencia anticristiana de algunos de sus asesores más próximos ${ }^{77}$.

El silencio mostrado por Dionisio acerca de la responsabilidad de Valeriano en la persecución desencadenada contra los cristianos en el transcurso de su reinado, que parece ser una consecuencia de su retórica griega tradicional, no logra eximir al emperador de su parte de responsabilidad en dicha fase persecutoria ${ }^{78}$.

Los consejos imperiales habían desempeñado ya un papel significativo en las persecuciones anteriores, de manera que su influencia sobre los diferentes emperadores parece haber sido decisiva en cuanto a la suerte de la Iglesia con anterioridad a dicho periodo: así, por ejemplo, sabemos que la atención de Nerón seria movida contra los cristianos, inmediatamente después del incendio de Roma, directamente por parte de su circulo de consejeros ${ }^{79}$.

De la misma manera el edicto promulgado por Septimio Severo, mediante el cual se prohibia la conversión al cristianismo, no se hubiera

74 Euseb., HE 7.10.4. Ver igualmente 7.10.5-8.

75 HE 7.10.4.

76 A. ALFÖLOI, "Zu den Christenverfolgungen in der Mitte des 3. Jahrhunderts", p. 339.

77 J. Daniélou y H. Marrou, Nouvelle histoire de l'Église, Paris 1963, 1, pp. 239-240.

78 P. KERESZTES, "Two Edicts of the Emperor Valerian", p. 89.

79 H. HEUBneR, "Zu Tac., Ann. 15,44,4", H 87 (1959), 223 y ss., y H. Fuchs, “Der Bericht über die Christen in den Annalen des Tacitus", VChr 4 (1950), 65 y ss. 
producido probablemente nunca sin la influencia de una fuerte camarilla anticristiana que rodeaba al emperador ${ }^{80}$.

Por ello el hecho de que Macriano se viera envuelto de manera directa en esta persecución no sólo no resulta increible sino que parece muy probable, y dicha implicación no le es atribuible a causa de su posición de procurator summarum rationum (procurador del patrimonio imperial) ${ }^{81}$, de igual manera que la finalización de este periodo de persecución en el transcurso del reinado de Galieno no debe ser asignada a Aurelio Quirinio, sucesor de Macriano en este elevado puesto de las finanzas ${ }^{82}$.

La situación casi desesperada por la que estaba atravesando el Imperio en distintos aspectos de su organización pudo contribuir sin duda al desarrollo de una situación apropiada para que Macriano y otros consejeros imperiales incidieran sobre Valeriano con respecto a las medidas a tomar contra los cristianos: la inacabable lista de calamidades e infortunios imperiales, así como la amenaza de destrucción que parecía cernirse sobre el Imperio, en especial en las fronteras orientales ${ }^{83}$, unido a la situación extremadamente caótica de la economía romana, así como a la todavia vigente plaga destructora y otra serie de desgracias, constituian factores que parecian hallarse fuera del control que podía imponer el gobierno romano ${ }^{84}$.

Ante tan desesperada situación no parecería extraño que el emperador Valeriano, al igual que habian hecho ya algunos de sus predecesores, como Marco Aurelio por ejemplo ${ }^{85}$, volviera su mirada hacia medidas de esta naturaleza, tratando de hallar en ellas un recurso contra lo sobrenatural, oculto o incluso mágico. En consecuencia, las acciones tomadas posteriormente contra los cristianos nos hacen pensar en ellos como las personas objeto de culpabilidad, teniendo presente que aparecerían a un mismo tiempo como víctimas propiciatorias con relación a la grave situación del Imperio.

Así pues, el primero de los edictos de Valeriano se nos presentaría cargado de unas características bastante inofensivas; el desarrollo de sus

80 P. Keresztes, "The Emperor Septimius Severus: a Precursor of Decius", Historia 19 (1970), 572-574.

${ }^{8}$ A. ALFÖLDI, " $Z$ U den Christenverfolgungen in der Mitte des 3. Jahrhunderts», p. 340.

82 Euseb., HE 7.13.1

83 A. AlFöLDI, “Die Hauptreignisse der Jahre 253-261 n. Chr. im Orient im Spiegel der Münzpraägung", Studien zur Geschichte der Weltkrise des 3. Jahrhunderts n. Chr., Darmstadt 1967, pp. 123 y ss.

84 Ver, por ejemplo, G. ALFöLdy, "The Crisis of the Third Century as seen by Contemporaries", GRBS 15 (1974), 89 y sS., y S. MROZEK, "Contribución al problema de la crisis financiera del Imperio romano en el siglo III d.C.(en polaco)», Przegl. Hist. 59 (1963), 470 y ss.

85 G. JOSSA, "Marco Aurelio e i cristiani", Giudei, pagani e cristiani, Nápoles 1977, pp. 109 y ss. 
disposiciones resulta muy claro a partir de la documentación antigua que nos ilustra sobre ello ${ }^{86}$. En contrapartida sus resultados parecen haber sido enormemente decepcionantes para el gobierno y administración romanos; además, todas las noticias de la época inciden en el hecho de que la Iglesia se había mostrado muy preparada para la persecución durante los años de vigencia de dicho edicto: tanto los obispos como el clero asumirian con entereza y buena disposición de ánimo su envio al destierro, lo que se muestra evidente en los casos de Cipriano y Dionisio de Alejandría, quienes únicamente se verían obligados a soportar ciertas incomodidades, entre ellas la de verse alejados de sus fieles ${ }^{87}$.

A pesar de todo los obispos y clérigos de Nubia (y Numidia) no se vieron tan favorecidos como sus correligionarios de Africa y Egipto: su suerte no consistiría simplemente en el cambio del confort de sus propios lugares de asentamiento por otros menos agradables a causa del exilio, puesto que el gobernador de Numidia condenaría a las minas a un buen número de los integrantes de este episcopado y clero con la finalidad de que soportasen todos los horrores y castigos que se hallaban implícitos en las deportaciones de esta naturaleza ${ }^{88}$.

Sin embargo, la comunidad cristiana del Imperio parece haber resultado por lo general bastante favorecida en su conjunto: a pesar del destierro de ciertos obispos y sacerdotes, asi como de la prohibición de celebrar asambleas y visitar los cementerios cristianos, la vida de las comunidades cristianas proseguiría casi en las mismas condiciones que con anterioridad. En realidad los sufrimientos de las victimas y el peligro que pendía sobre los cristianos parece haber servido a éstos de inspiración: así, en Egipto, Africa y Numidia se continuarían celebrando asambleas, mientras que los fieles siguieron cumpliendo su culto y ritos, y los prisioneros fueron visitados y ayudados ${ }^{89}$.

Por consiguiente este primer decreto de Valeriano contra los cristianos promovería una persecución esencialmente incruenta: la forma de hacer frente a dichas circunstancias por parte de la jerarquia eclesiástica y los laicos constituye un triunfo evidente, ocasionando como contrapartida el fracaso de esta medida imperial.

96 P. KeRESzTES, "Two Edicts of the Emperor Valerian", p. 90.

L. LuDwIG, Der heilige Martyrerbischof Cyprian von Karthago, Munich 1951.

88 J.G. DAVIES, "Condemnations to the Mines...", Univ. Birmingham Historical Journal 6 (1958), 99 y ss.

Eq Euseb., HE 7.11.12-14. Cf. Cypr., Epist. 76-79... 
Habiéndose purificado a través de dicho fracaso en el transcurso de la persecución de Decio ${ }^{90}$, los cristianos se encontrarían mucho más preparados para morir por su fe en los años siguientes que lo habian estado con anterioridad; de esta manera, en el momento en que se promulgó el segundo sangriento edicto, Cipriano y sus compañeros contarian ya con una buena disposición de ánimo con respecto a él, poseyendo al mismo tiempo un conocimiento bastante adecuado en lo que se refería al alcance, avance y desarrollo que podía tomar.

Ante los resultados catastróficos alcanzados por el primero de dichos edictos, el recurrir al segundo de ellos parece haber constituido un paso en cierto modo lógico: si los objetivos del primer decreto consistieron en paralizar la organización y funcionamiento interno de la Iglesia cristiana ${ }^{91}$, las medidas emanadas del segundo edicto parecen haber servido mucho mejor a este mismo fin a causa del carácter eminentemente práctico que las animaba, buscando con ello una liquidación total, y no sólo el destierro, de los miembros de la jerarquía cristiana.

Sin embargo, este segundo decreto se propondría ir más allá de la simple liquidación de los ministros cristianos: si observamos con detenimiento las disposiciones del mismo en el relato de Cipriano, parece desprenderse de él que se trataria de medidas puramente fiscales ${ }^{92}$.

De este modo, mientras no parecen deducirse del primer decreto (o lo son en muy pequeña medida) estas consideraciones financieras, la confiscación de las fortunas privadas se convertiria en uno de los objetivos prioritarios en el segundo. Igualmente, mientras que no podemos hallar una confirmación explicita con respecto a los bienes personales ni en las Actas ni en las Pasiones más significativas, las referencias a la liquidación por parte de Galieno de la persecución edictada por su padre constituyen una evidencia palpable en cuanto a las confiscaciones del patrimonio de la Iglesia ${ }^{93}$.

90 Ver, entre otros, Ch. SAUMAGNE, "La persécution de Dèce en Afrique d'après la correspondence de saint Cyprien", Byzantion 32 (1962), 1 y ss.

${ }^{9}$ J. MOLThAGEN, Der römische Staat und die Christen im zweiten und dritten Jahrhundert, pp. 87-92

92 H. GREgolRe y otros, Les persécutions dans l'empire romain, p. 50.

93 Euseb., HE 7.13. Cf. W.H.C. FREND, “Wich Dionysius?. Eusebius, HE VII,13", Latomus 36 (1977), 164-168. 
En este mismo sentido las puntualizaciones añadidas por Cipriano en sus referencias al edicto pueden implicar igualmente la confiscación de bienes, no sólo los pertenecientes a la comunidad eclesiástica sino también los propios de los cristianos en cuanto personas particulares ${ }^{94}$.

De todo ello se desprende que en la base, contenido y medidas emanadas de este segundo documento de Valeriano se hace patente que la motivación económica sería tomada muy en serio por parte de las autoridades romanas para el caso de dicha persecución ${ }^{95}$. Sin duda la grave situación económica que atenazaba al Imperio pudo haber constituido un elemento a considerar desde el punto de vista de que los nuevos edictos imperiales pondrian el énfasis en la solución de dicha crisis.

De este modo, aunque las plagas que seguían mostrándose aún violentas en las provincias romanas, habian contribuido, al menos de forma indirecta, a las persecuciones anteriores, su influencia permanece oscura en tiempos de Valeriano: en el momento de la entrada en vigor de las nuevas medidas imperiales contra los cristianos la economía romana atravesaba por unos momentos extremadamente críticos, puesto que la inflación era muy seria y además la moneda imperial había pasado a ser algo ya totalmente inservible ${ }^{96}$.

A todo ello hemos de añadir el hecho de que el erario público se veria completamente debilitado como consecuencia de la pérdida de los impuestos, tanto en las provincias orientales como en las occidentales del Imperio.

Se ha venido suponiendo, por otra parte, que la Iglesia, desde el punto de vista económico, constituiría una especie de oasis en el marco de la reseca economía del Imperio; en este sentido parece cierto que las comunidades cristianas en general ( $y$ la Iglesia en su conjunto) eran relativamente prósperas y que en su seno se incluía un grupo de individuos bastante ricos ${ }^{97}$. La cuestión estriba en conocer si el innegable patrimonio común y privado de que disponía la Iglesia cristiana proporcionó un motivo económico, directo o indirecto, para el desencadenamiento de la persecución de Valeriano en su segunda fase, ya que resulta muy poco probable que existiera una motivación económica detrás del primero de sus decretos ${ }^{98}$.

94 Cypr., Epist 80.1 y 4. Cf. P. KeRESzTES, "Two Edicts of the Emperor Valerian", p. 91.

95 G.T. OBORN, "Why did Decius and Valerian proscribe Christians?", ChHist 2 (1933), 67 y ss.

96 Remitimos sobre estos problemas, entre otros, a A. ALFöLDI, "La grande crise du monde romain au Ille siècle", $A C 7(1958), 1$ y ss., y G. ALFÓLOY, "Der heilige Cyprien und die Krise des römischen Reiches", Historia 22 (1973), 36 y ss.

97 P. KeRESzTES, "Two Edicts of the Emperor Valerian", p. 92.

98 J. MOLTHAGEN, Der römische Staat und die Christen im zweiten und dritten Jahrhundert, p. 86. 
Las propiedades de la comunidad eclesiástica parecen haber permanecido intactas durante los anos de la primera etapa persecutoria de Valeriano, sobre todo si tenemos presentes las medidas del edicto que la provocó, que prohibian las asambleas y visitas a los cementerios; además, no contamos con ninguna noticia acerca de posibles confiscaciones llevadas a cabo como consecuencia de ello.

En cuanto a las consideraciones financieras que aparecen reflejadas en el segundo edicto se nos muestran extremadamente explícitas: de acuerdo con la visión presentada por Cipriano sobre dicho decreto las confiscaciones de bienes parecen haber sido más abundantes que las ejecuciones ${ }^{99}$. Por consiguiente la confiscación constituiría el castigo previsto para todas las personas relacionadas con la religión cristiana si hacemos excepción de los miembros integrantes de la jerarquía eclesiástica.

En este sentido el edicto parece mostrarse particularmente duro con los empleados de palacio y de la administración imperial (Caesariani), puesto que quienes de ellos hubieran confesado ya o confesaran su fe en estos momentos perderían sus propiedades a través de la confiscación ${ }^{100}$. Sin embargo, esta frase de Cipriano parece suscitar ciertos interrogantes aun sin resolver: ¿no se les daría la oportunidad, de acuerdo con lo que había sido norma general hasta entonces, de poder negar su condición de cristianos?; ¿qué momento concreto de la persecución aparece reflejado en dicha frase?...

Una situación similar de oscuridad es la que encontramos con respecto a los senadores, los personajes de posición elevada y los ciudadanos romanos pertenecientes al orden ecuestre, a quienes parece habérseles negado la posibilidad de renunciar al cristianismo; todas estas personas perderían su rango social y patrimonio, siendo castigadas con su propia vida en el caso de que perserverasen en su fe cristiana ${ }^{101 .}$

Por su parte las matronas romanas se verian obligadas a soportar medidas parecidas: aunque sus vidas no llegarían a estar realmente en peligro, no se les daria la oportunidad de elegir entre el cristianismo o la pérdida de sus bienes acompañada del destierro. Si lo descrito por Cipriano sobre el castigo impuesto a todos estos personajes laicos cristianos resulta cierto, hemos de concluir que el segundo decreto de

39 H. GRÉGOIRE y otros, Les persécutions dans l'empire romain, p. 49.

100 Cypr., Epist. 80.1 y 2. Cf. G.W. CLARKE, "Prosopographical Notes on the Epistles of Cyprian, III", pp. 443-444.

101 J. PLESCIA, "On the Persecution of the Christians in the Roman Empire", Latomus 30 (1971), 126. 
Valeriano pondria un énfasis inusual en desposeer a tales personas de sus bienes ${ }^{102}$.

Sin embargo, aun en el caso de que las medidas anticristianas contenidas en el segundo edicto, de acuerdo con el relato de Cipriano, sean verdaderas, la puesta en práctica de las mismas plantea una historia algo diferente. así, por ejemplo, el documento imperial prescribe que los obispos, sacerdotes y diáconos fueran castigados con la muerte de inmediato. Ahora bien, si echamos una ojeada a las Actas y Pasiones correspondientes a estos años observaremos que los integrantes de la jerarquía eclesiástica únicamente serian condenados a muerte tras la celebración de juicios regulares, en los que se les concedía la oportunidad de negar su condición de cristianos, ofrecer sacrificios a los dioses romanos y quedar libres a continuación ${ }^{103}$.

Entre las Pasiones contamos con un ejemplo que hace cuestionable la exactitud del relato de Cipriano: la Pasión de los santos Mariano y Santiago hace mención de un personaje de rango ecuestre, de nombre Emiliano, quien, tras la confesión de su fe cristiana, seria condenado a muerte por parte del legado de Numidia ${ }^{104}$.

Las circunstancias en las que los enviados de Cipriano parecen haber recogido su información en Roma estuvieron envueltas sin duda en una situación de completo pánico; a pesar de ello el obispo de Cartago considera dichos relatos como verdaderos, aunque admite que existían abundantes y contradictorios rumores con respecto al rescripto enviado por Valeriano al Senado acerca de la muerte de los cristianos ${ }^{105}$.

Igualmente hace referencia Cipriano a las cartas enviadas por el emperador a los gobernadores provinciales: en este sentido el documento enviado al delegado del gobierno romano en Africa constituye la base en que se apoya dicho autor para sus conclusiones (resulta sumamente improbable que el contenido de la carta remitida a los gobernadores fuera distinto de la realidad expresada en el documento enviado al Senado).

Por lo tanto la confiscación de las propiedades comunitarias de la Iglesia, unido a toda una serie de confiscaciones de las fortunas privadas, pudo convertir al erario público en más rico; sin embargo, si tenemos en cuenta la gravedad de la crisis financiera en la que se hallaba sumido el

102 Cypr., Epist. 80.1. Cf. W.H.C. FREND, Martyrdom and Persecution in the Early Church, p. 426.

103 P. KERESZTES, “Two Edicts of the Emperor Valerian", p. 93.

104 Passio sanctorum Mariani et lacobi 8-10 y 34.

105 Cypr., Epist. 80.1. 
Imperio en esta época ${ }^{106}$, la ayuda prestada al tesoro imperial por parte de estas confiscaciones de los bienes cristianos seria inapreciable. En consecuencia, hemos de desechar la idea de que el motivo más importante que se halla implícito en el segundo edicto de persecución anticristiana de Valeriano lo constituiría el reaprovisionamiento del tesoro y admitir, sin embargo, que la destrucción de la Iglesia, o mejor dicho de su organización y de las prácticas de vida cristiana pueden ser consideradas como el objetivo fundamental de ambos decretos imperiales ${ }^{107}$.

El primero de tales edictos no contendría, o lo haría de forma muy débil, ningún motivo económico destacable; su objetivo más inmediato consistiría en el desmantelamiento de la organización y culto cristianos (la prohibición de celebrar asambleas y visitar los cementerios bajo la amenaza de castigos corporales subraya esta finalidad destructiva).

Por su parte el segundo de tales decretos añadiría a estas medidas otras que completarían el desmembramiento y desarbolamiento de la organización de la Iglesia cristiana, puesto que no dejaba en libertad a los integrantes del clero hasta que no morían o apostataban ${ }^{108}$. La eliminación de los altos personajes de la jerarquía cristiana significaría que no formarían parte ya del Senado ni de los cuerpos y puestos más elevados de la sociedad y administración romanas, pero también que la Iglesia perdería su principal fuente de ingresos.

La razón que explicaría el deseo de abolir la organización eclesiástica estribaría en el hecho de que Valeriano habría sido convencido de que la Iglesia cristiana representaba un peligro para el Estado romano ${ }^{109}$ : la situación real por la que estaba atravesando el Imperio en estos momentos era sumamente trágica, por lo que no resultaría demasiado difícil convencer al emperador, que se tambaleaba en medio de un caos económico y militar, de que los cristianos deberian ser objeto de reprobación por parte del pueblo y del propio Senado romanos.

De esta manera no existe razón alguna para no creer en el relato de Dionisio de Alejandría, quien nos asegura que Macriano convencería al sugestionable emperador de que era preciso que la Iglesia cristiana fuese objeto de censura y posteriormente de destrucción ${ }^{110}$.

106 E. Albertini, L'empire romain, París 1970 (4ª edic.), pp. 302-309. Cf. J.P. Callu, La politique monétaire des empereurs romains de 238 à 311 , París 1969.

107 P. KereszTES, "Two Edicts of the Emperor Valerian", p. 94.

108 G.W. ClaRKE, "Prosopographical Notes on the Epistles of Cyprian, II", pp. 441-442.

109 Acta proc. s. Cypriani 4...

$1:$ H. GRÉgoIRE y otros, Les persécutions dans l'empire romain, p. 45. 